\title{
Simulation of Drastic Lag Phenomena in GaAs-Based FETs for Large Voltage Swing
}

\author{
K. HORIO*, Y. MITANI, A. WAKABAYASHI and N. KUROSAWA \\ Faculty of Systems Engineering, Shibaura Institute of Technology, \\ 307 Fukasaku, Omiya 330-8570, Japan
}

\begin{abstract}
Turn-on characteristics of GaAs MESFETs and HEMTs are simulated when the gate voltage is changed abruptly. The gate-lag or slow current transient becomes more pronounced when the off-state gate voltage is more negative, because the surface-state effects or substrate-trap effects become more significant. Changes of I - V curves of GaAs MESFETs, when the drain voltage is swept with different speeds, are also simulated. When the swept time is short, the curve shows overshoot-like behavior and the kink disappears, indicating that the $\mathrm{I}-\mathrm{V}$ characteristics should be quite different between DC and RF conditions.
\end{abstract}

Keywords: GaAs MESFET; HEMT; Gate-lag; Drain-lag; Trap; Kink phenomena

\section{INTRODUCTION}

In GaAs MESFETs and HEMTs, slow current transients are often observed experimentally even if the drain voltage or the gate voltage is changed abruptly. They are called "drain-lag" or "gatelag" and could be fatal when the high-speed and high-frequency operation is considered [1-3]. As for factors of these slow transients, effects of deep traps in the semi-insulating substrate and surface states on the semiconductor surface are suggested, but the detailed mechanisms are not well clarified. And hence, there is no definite way established to eliminate them. Here, it should be pointed out that few theoretical works are reported which treat these phenomena.
Therefore, in this work, we have made transient simulations of GaAs MESFETs and AlGaAs/ GaAs HEMTs when the gate voltage or the drain voltage is swung significantly, and showed that the lag phenomena become very pronounced for larger changes of the voltages. This indicates that the $\mathrm{I}-\mathrm{V}$ characteristics should be quite different between DC and RF conditions and the lag phenomena should be considered in the modeling of GaAs-based power devices.

\section{PHYSICAL MODEL}

Here, we consider recessed-gate FETs as well as normal planar FETs. Figure 1 shows device

*Corresponding author. e-mail: horio@sic.shibaura-it.ac.jp 


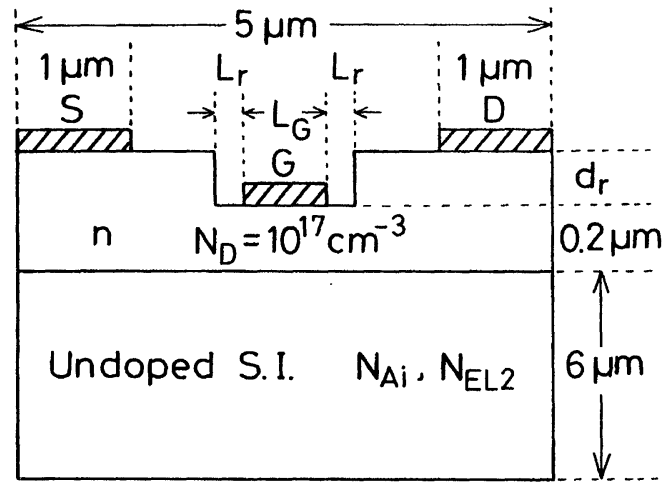

(a)

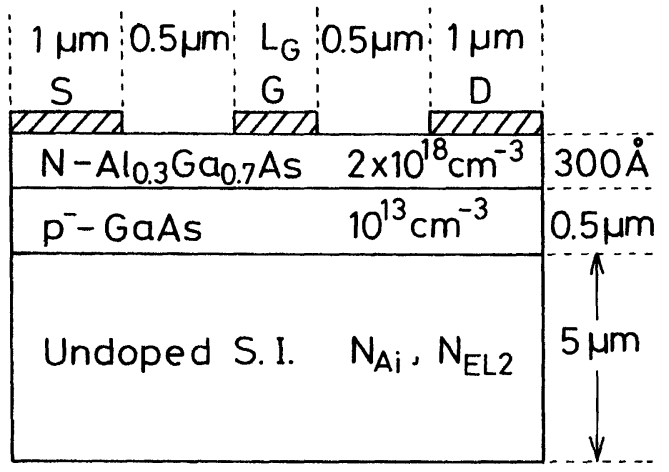

(b)

FIGURE 1 Device structures simulated in this study. (a) Recessed-gate GaAs MESFET, (b) modeled AlGaAs/GaAs HEMT.

structures analyzed here. Figure 1a is a recessedgate GaAs MESFET, and Figure $1 \mathrm{~b}$ is a modeled AlGaAs/GaAs HEMT. For a substrate, we consider undoped semi-insulating LEC GaAs where deep donors "EL2" $\left(N_{E L 2}\right)$ compensate shallow acceptors $\left(N_{A i}\right)$. As for a surface-state model, we adopt Spicer's unified defect model [4], and assume that the surface states consist of a pair of deep donor and deep acceptor. Their densities are typically set to $10^{13} \mathrm{~cm}^{-2}$. As to their energy levels, the following case based on experiments is considered: $E_{S D}=0.87 \mathrm{eV}$ and $E_{S A}=0.7 \mathrm{eV}[3,5]$, where $E_{S D}$ is the energy difference between the bottom of conduction band and the surface deepdonor's energy level, and $E_{S A}$ is the energy difference between the surface deep-acceptor's energy level and the top of valence band. Basic equations to be solved are Poisson's equation including ionized deep-level terms, continuity equations for electrons and holes which include electron and hole loss rates via the deep levels and a carrier generation rate by impact ionization, and three rate equations for the deep levels. These equations are put into discrete forms, and are sloved numerically. It should be mentioned that the drift-diffusion model is used here, and the results given below may be affected by the nonequilibrium carrier transport on the scale of $10^{-12} \sim 10^{-11} \mathrm{~s}$.

\section{GATE-LAG PHENOMENA}

First, we have calculated trun-on characteristics of GaAs MESFETs and HEMTs when the gate voltage is switched on abruptly. Usually, the OFF state is set so that the DC drain current becomes $5 \times 10^{-3} \mathrm{~A} / \mathrm{cm}$. In this case the gate voltage at the OFF state $V_{\text {Goff }}$ corresponds to the threshold (pinch-off) voltage $V_{t h}$. In the power devices, the gate voltage can be more negative than $V_{t h}$, so that we have also calculated the $V_{\text {Goff }}$ dependence.

Figure $2 \mathrm{a}$ shows an example of calculated turnon characteristics of a recessed-gate GaAs MESFET as a parameter of $V_{\text {Goff. When } V_{\text {Goff }} \text { is }}$ equal to $V_{t h}$, the drain current remains a low value for some periods and begins to increase slowly, showing the gate-lag behavior. This occurs due to the slow response of surface states. When $V_{\text {Goff }}$ is much more negative than $V_{t h}$, the gate-lag becomes more significant because the surface-state effects are more pronounced to widen the surface depletion layer at the OFF state. It is also seen that the drain current shows overshoot-like behavior at relatively early periods. This is understood due to the substrate-trap effects [6]. For strongly negative $V_{G o f f}$, electron depletion occurs also in the semiinsulating substrate, and electrons are injected into the substrate during turn-on, leading to the overshoot-like behavior. These two observations for 

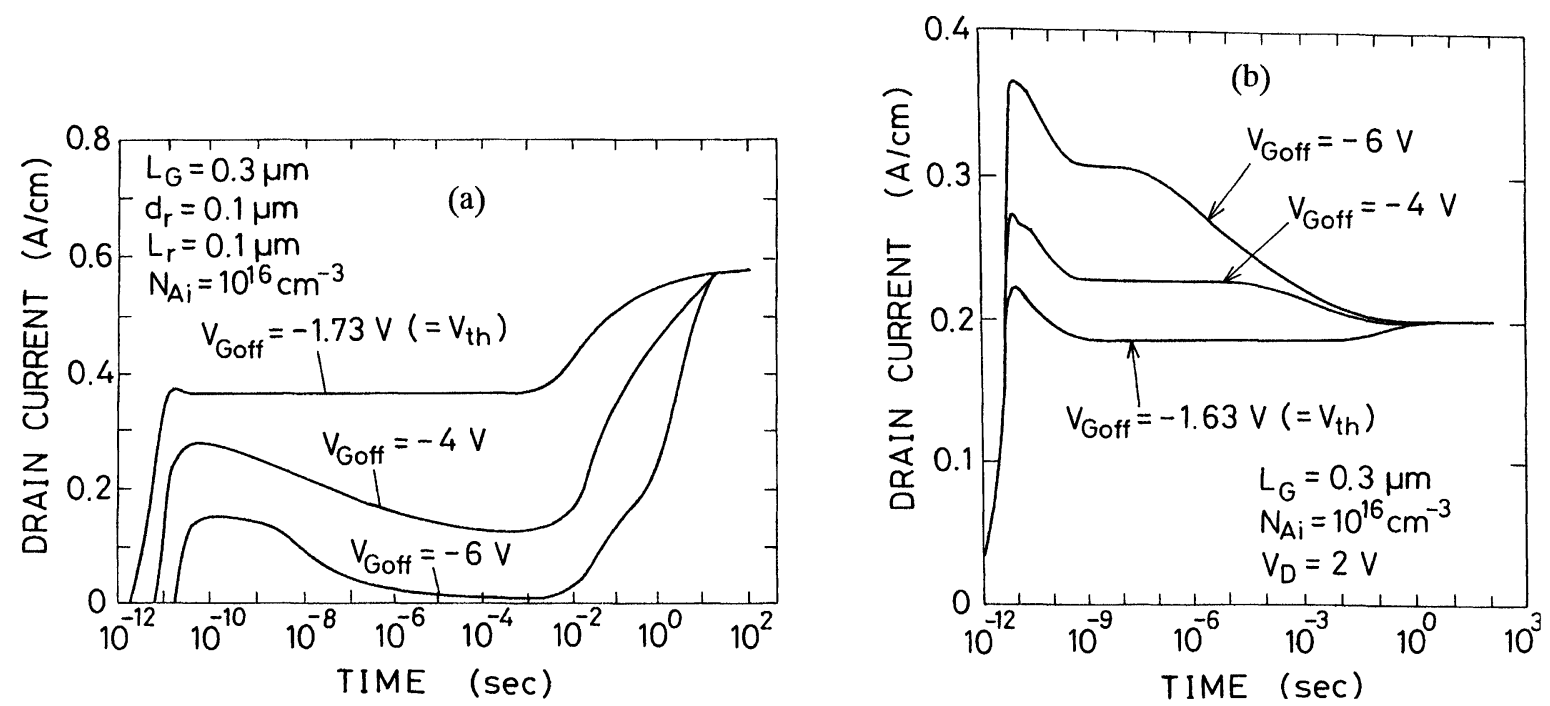

FIGURE 2 Calculated turn-on characteristics of a recessed-gate GaAs MESFET (a) and an AlGaAs/GaAs HEMT (b) as a parameter of the off-state gate voltage $V_{G o f f}$.

strongly negative $V_{\text {Goff }}((1)$ more pronounced gatelag and (2) overshoot-like behavior) are qualitatively consistent with published experimental data for a recessed-gate GaAs MESFET [2]. The above results indicate that when the device is kept at the OFF state, dynamic $I-V$ characteristics should become quite different from the steady-state ones.

Figure $2 \mathrm{~b}$ shows calculated turn-on characteristics of an $\mathrm{AlGaAs} / \mathrm{GaAs}$ HEMT as a parameter of $V_{\text {Goff }}$, where the surface-state effects may not be so important. When $V_{\text {Goff }}$ is much more negative than $V_{t h}$, abnormal current overshoot and subsequent slow transients are observed. These can be understood from the substrate-trap effects as in GaAs MESFETs.

\section{DRAIN-LAG AND DYNAMICS OF I-V CURVES}

Next, we describe the dynamics of GaAs MESFETs when the drain voltage is changed. Figure 3 shows the responses of drain currents when the drain voltage $V_{D}$ steps abruptly from 0 to $1 \mathrm{~V}$ or from 0 to $13 \mathrm{~V}$ while keeping the gate voltage $0 \mathrm{~V}$. For $V_{D}=0$ to $1 \mathrm{~V}$, the drain current remains a slightly high value for some time and begins to decrease gradually (around $10^{-5} \mathrm{~s}$ ), reaching the real steady-state value. For the large change of $V_{D}$ ( 0 to $13 \mathrm{~V})$, the drain current shows remarkable overshoot and begins to decrease earlier (around $10^{-9} \mathrm{~s}$ ). These overshoots occur because electrons are injected into the substrate when $V_{D}$ is raised, and the deep traps (EL2) in the substrate need certain time to capture these electrons. For larger change of $V_{D}$, more electrons are injected into the substrate, and hence the overshoot becomes more remarkable, and the drain current begins to decrease earlier because the capturing time by the traps is inversely proportional to the electron density.

Figure 4 shows calculated $I_{D}-V_{D}$ curves when the drain voltage is swept from 0 to $13 \mathrm{~V}$ with different speeds. The parameter is the swept time $\tau$. When $\tau$ is short, the drain currents become much higher than the steady-state values, because EL2 needs certain time to capture injected electrons. Another interesting point is that kink behavior arises in the steady-state $\mathrm{I}-\mathrm{V}$ curve for a case with 


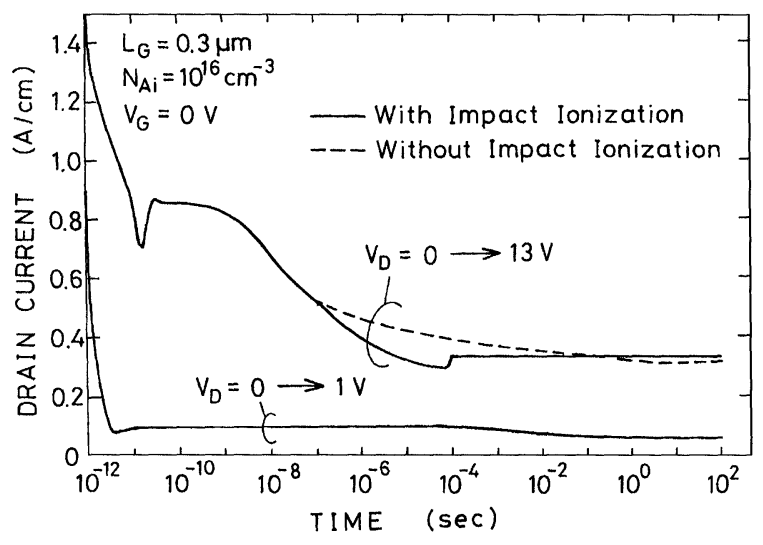

FIGURE 3 Responses of drain currents of a GaAs MESFET when the drain voltage changes abruptly from 0 to $1 \mathrm{~V}$ or from 0 to $13 \mathrm{~V}$ while keeping the gate voltage constant.
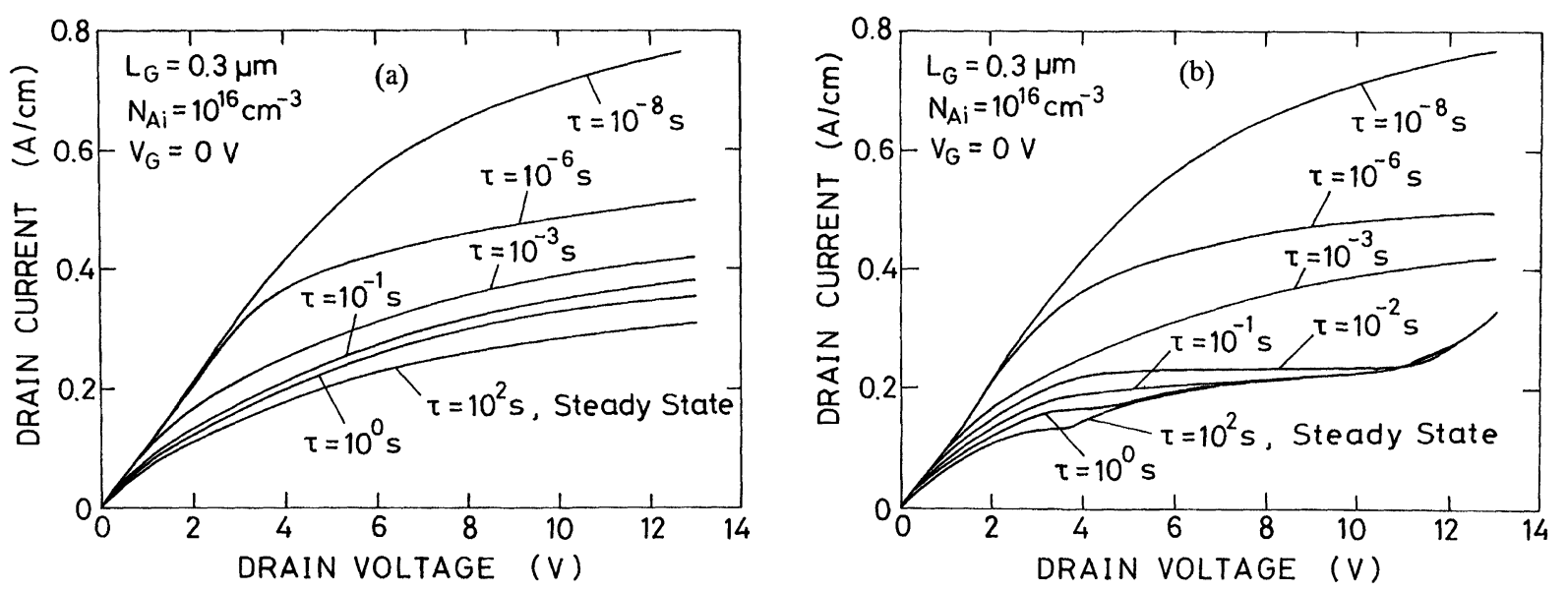

FIGURE 4 Calculated $I_{D^{-}} V_{D}$ curves of the GaAs MESFET when the drain voltage is swept from 0 to $13 \mathrm{~V}$ with different speeds. $\tau$ is the swept time. (a) Without impact ionization, (b) with impact ionization.

impact ionization, and the kink disappears when $\tau$ is short. This indicates that the kink, which is originated from the interaction between generated holes by impact ionization and the substrate traps [7], is a rather slow process. The above results indicate that $\mathrm{I}-\mathrm{V}$ characteristics of $\mathrm{GaAs}$ MESFETs should be quite different between $\mathrm{DC}$ and RF conditions.

\section{CONCLUSION}

Two-dimensional transient simulations of GaAs MESFETs and AlGaAs/GaAs HEMTs are performed when the gate voltage or the drain voltage is changed abruptly. The gate-lag and the drain-lag are reproduced, which are explained by the dynamics of surface states and deep levels in the 
substrate. The lags becomes more significant for larger changes of the voltages. It is concluded that the lag phenomena should be considered in the modeling of GaAs-based power devices.

\section{References}

[1] Yeats, R. et al. (1988). "Gate slow transients in GaAs MESFET's - Causes, cures, and impact on circuits", IEDM Tech. Dig., pp. 842-845.

[2] Kohno, Y. et al. (1994). "Modeling and suppression of the surface trap effect on drain current frequency dispersions in GaAs MESFETs", Proc. GaAs IC Symp., pp. 263-266.
[3] Horio, K. and Yamada, T. (1999). "Two-dimensional analysis of surface-state effects on turn-on characteristics in GaAs MESFET's", IEEE Trans. Electron Devices, 46, $648-655$.

[4] Spicer, W. E. et al. (1979). "New and unified model for Schottky barrier and III-V insulator interface states formation", J. Vac. Sci. Technol., 16, 1422-1433.

[5] Wieder, H. H. (1983). "Surface Fermi level of III-V compound semiconductor-dielectric interfaces", Surface Sci., 132, 390-405.

[6] Horio, K., Wakabayashi, A. and Yamada, T. (2000). "Two-dimensional analysis of substrate-trap effects on turn-on characteristics in GaAs MESFET's", IEEE Trans. Electron Devices, 47, 617-624.

[7] Horio, K. and Satoh, K. (1994). "Two-dimensional analysis of substrate-related kink phenomena in GaAs MESFET's", IEEE Trans. Electron Devices, 41, 2256-2261. 

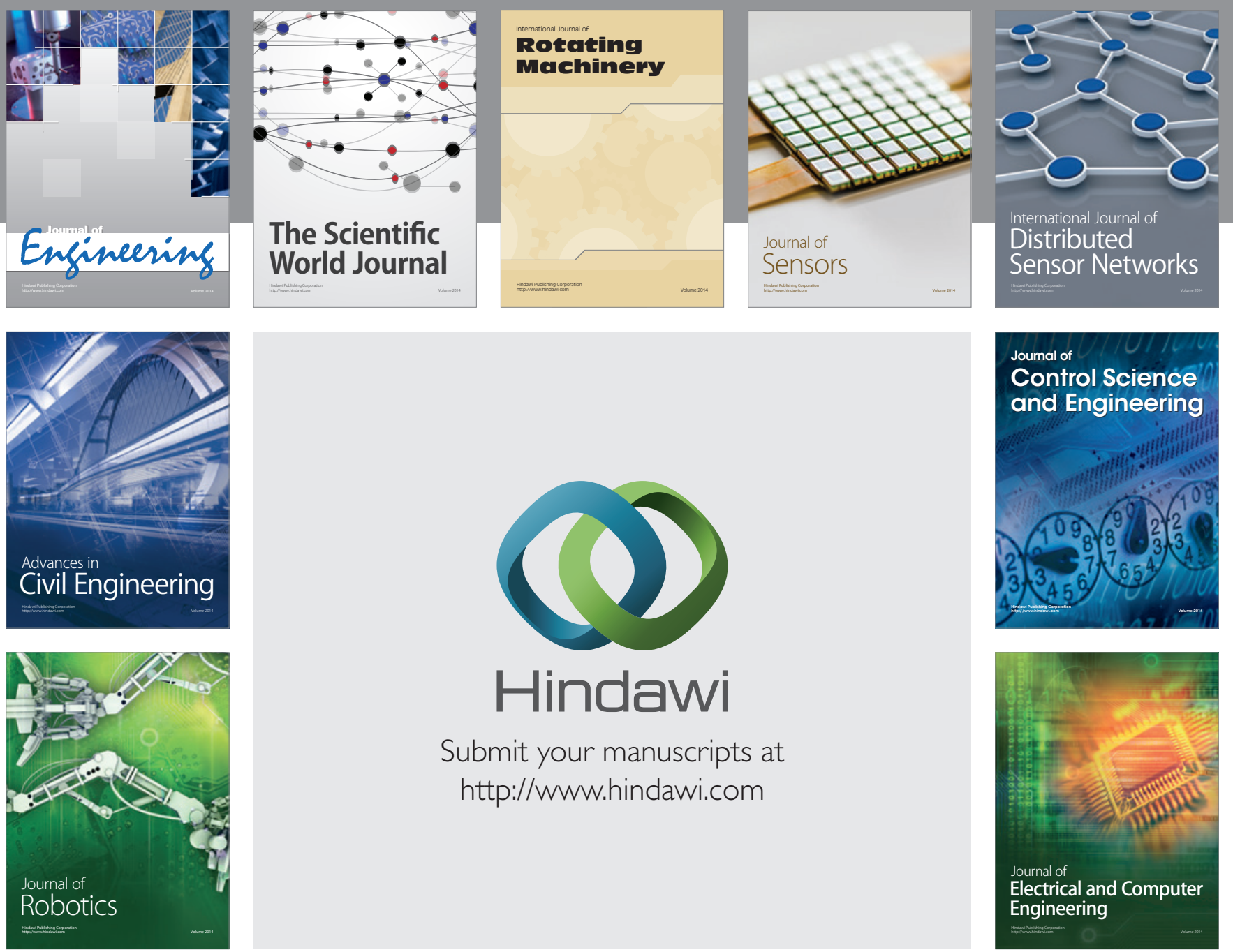

Submit your manuscripts at

http://www.hindawi.com
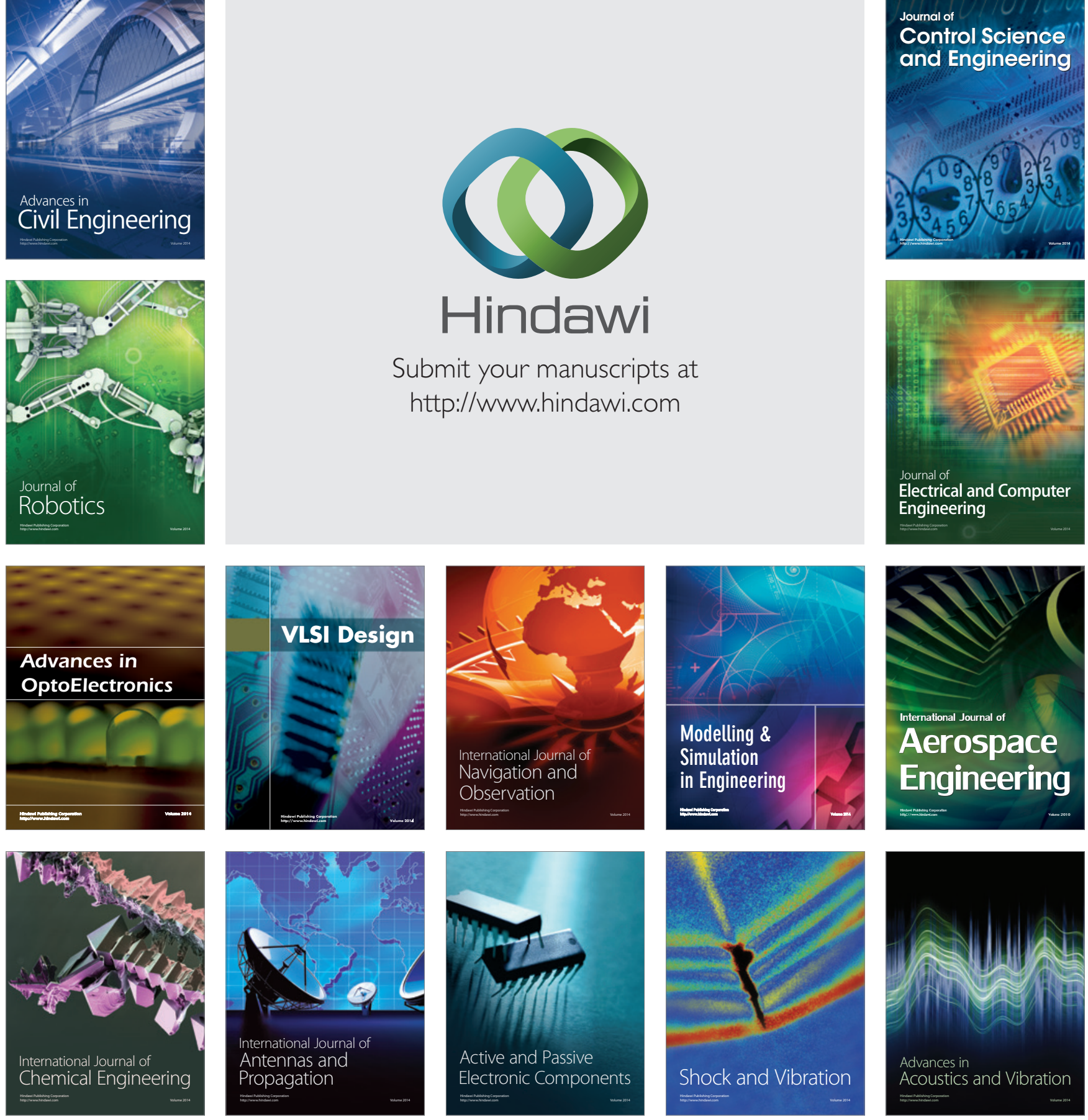